\title{
E34 UPSCALING OF PERMEABILITY IN THE WELL VICINITY ON DISTORTED GRIDBLOCKS
}

Institut Français du Pétrole, 1 \& 4 Av. Bois-Préau, 92852 Rueil Malmaison, FRANCE

\begin{abstract}
Upscaling of absolute permeability has been long standing discussed. It is generally recognized that the main difficulty in permeability upscaling is the dependence of upscaling results on flow boundary conditions, which leads to a non-unique solution. However, a suitable solution can be found for accurate upscaling in the well vicinity, because flow in the near-well region is dominated by the well driven flow and upscaling results are almost independent on other boundary conditions. In this paper, near-well upscaling techniques are discussed, and we present a method for near-well upscaling for advanced wells on 3D CPG (Corner-Point Geometry) gridblocks. With the proposed near-well upscaling procedure, accurate near-well flow behavior can be obtained with coarse CPG grid simulation. Examples show equivalent results on well performance with coarse grid and fine grid simulations.
\end{abstract}

\section{Introduction}

Upscaling of permeability from geological/geostatistical model to reservoir flow simulation model has been discussed for a long time (cf. [2], [10]). The widely used upscaling methods are based on solving a steady-state flow equation on fine gridblocks and determining equivalent parameters on corresponding coarse gridblocks using the solution of fine grid simulation. However, the upscaling results depend greatly on boundary conditions for fine grid flow simulation (cf. [2], [10], [16]). This leads to non-unique upscaling solution. Different upscaling procedures might give very different results. It is difficult to conclude one method is better than another, because neither boundary conditions used in upscaling procedure correspond really to flow patterns in a real field case.

The aim of upscaling is to provide block permeabilities for coarse grid flow simulations. For a field problem, reservoir simulators are mainly used to investigate well productivities, such as oil production rate, wateroil ratio, etc. These data are accessed from the well. So, accurate flow simulation in the well and near the wellbore is very important. For the same reason, having an accurate upscaling procedure in the near-well region is also very important. Accurate near-well upscaling can improve dramatically simulation accuracy for well productivities in heterogeneous media.

The first near-well upscaling procedure is proposed by Ding (cf. [3]) in 1995. In that paper, the author illustrated the necessary and the importance of a particular treatment for permeability upscaling in the well vicinity. Ding (cf. [3]) developed an approach that entails the solution of a single-phase, well driven flow problem. This solution is then used to determine near-well block transmissibilities in addition to the well index. A clear improvement was demonstrated with this technique over standard approaches (cf. [3], [5]). The success of using near-well upscaling in flow simulations is that the flow pattern in the well vicinity is well defined and the boundary condition for fine grid simulation is controlled by the presence of the well. This concept of near-well upscaling have then been developed for various applications. Durlofsky et al. (cf. [8]) used the near-well upscaling in a field application. Mascarenhas and Durlofski [12] and Muggeridge et al. [13] applied this approach to horizontal wells. At the present, all developments for near-well upscaling are limited to Cartesian coarse gridblocks. However, in real applications of flow simulation, CPG (Comer-Point Geometry) gridblocks are greatly used. So, it is necessary to study near-well upscaling techniques on distorted gridblocks.

Upscaling on CPG gridblocks is more difficult because of grid distortion and no superposition between coarse gridblocks and fine gridblocks (cf. [17]). However, in the well vicinity, like all the near-well upscaling procedures, flow pattern is dominated by the presence of the well. The difficulty of dependence on boundary conditions is minimised in near-well region by using well driven flow simulation. In section 2 , an analysis is given

$8^{\text {th }}$ European Conference on the Mathematics of Oil Recovery - Freiberg, Germany, 3 - 6 September 2002 
to discuss the problem of dependence of near-well upscaling results on boundary conditions. To overcome the problem of no superposition between coarse gridblocks and fine gridblocks, the marching cube method is used to calculate geometrical interception between gridblocks in 3D.

The objective of upscaling procedure is to provide the coarse grid flow simulation as close as to the fine grid one. So, the accuracy of scaling-up results depends on the accuracy of the fine grid simulation. If the proposed upscaling procedure is validated, we can get an accurate well model on fine gridblocks in heterogeneous media. For instance, we can refine fine gridblocks around the well, so permeability variation is relatively homogeneous from block to block. Well model developed for homogeneous media (cf. [4]) can be used and considered accurate on refined fine gridblocks. Then, we apply the near-well upscaling from the refinement of the fine gridblocks to the fine gridblocks. So, suitable well model can be obtained for flow simulation on fine gridblocks with the near-well upscaling procedure. In this paper, we will not discuss the accuracy of fine grid simulation, and in fact, this problem can be solved if the proposed upscaling procedure is validated, as described in [3]

The proposed near-well upscaling procedure is developed for advanced wells on 3D CPG gridblocks. Some examples are presented. These examples show the efficiency and necessary of near-well upscaling. Using near-well upscaling procedures, simulation errors on well performance can be greatly reduced. The proposed procedure gives generally very satisfied results.

\section{Near-well upscaling procedure in $2 \mathrm{D}$}

Accurate flow simulation on coarse gridblocks depends not only on accuracy of upscaling procedure, but also on accuracy of numerical scheme used in flow simulation. To avoid the loss of accuracy in each step, we combine together the upscaling procedure and numerical schemes. In other word, we will upscale directly transmissibilities, the discretization coefficient in numerical schemes, instead of block permeabilities. The advantages of upscaling transmissibility have been recognized by numerous authors (cf. [15], [17]).

\section{Numerical schemes on distorted gridblocks in the well vicinity}

Multi-point flux approximation schemes (cf. [1], [9]) are generally used for flow modelling on distorted gridblocks. In the well vicinity, "radial" flow is usually dominating and the pressure variation follows a logarithmic behavior instead of a linear behavior. So, numerical schemes should also adapt to the particular near-well flow behavior, and this aspect is usually more important. An analysis for the near-well flow modelling was given in [7]. It was shown that a two-points scheme can be used to catch near-well behavior and gives generally satisfied results for well modelling.

A two-point flux approximation scheme can be written as follows:

$$
F_{i j}=-T_{i j}\left(p_{j}-p_{i}\right)
$$

where $F_{i j}$ is the flux across the interface between two gridblocks $\mathrm{i}$ and $\mathrm{j}, T_{i j}$ is the transmissibility term, and $p_{i}, p_{j}$ are pressures on gridblocks $\mathrm{i}$ and $\mathrm{j}$ (Figure 1). Although a two-point scheme is considered inaccurate for non orthogonal gridblocks, it is possible using it to obtain satisfied near-well simulation results. The key point is to determine the transmissibility coefficient $T_{i j}$ so that Eq. (1) is an accurate approximation for a singular near-well flow (cf. [7]).

In homogeneous media, we have presented an analytical formula for transmissibility calculation (cf. [7]). Transmissibility determination in heterogeneous media is the object of this work.

\section{Near-well upscaling procedure}

Transmissibility upscaling is based on a steady-state flow. Without loss of generality, we assume viscosity $\mu=1$ in upscaling procedures. Having a solution of a steady-state problem on fine gridblocks, equivalent coarse block permeabilities and flux on coarse gridblock interface can be calculated. Then, transmissibility on coarse gridblocks can be determined with an inverse procedure as follows (Figure 1) (cf. [3], [5]):

$$
T_{i j}=-\frac{F_{i j}}{p_{j}-p_{i}}
$$


where $F_{i j}$ represents the equivalent flux on coarse block interface, $p_{i}$ and $p_{j}$ represent equivalent coarse block pressures. To get a good equivalent block transmissibility, it is necessary having an accurate calculation for equivalent flux and equivalent pressures. Like all upscaling procedures, the technique presented above is usually boundary condition dependant. For different boundary conditions on fine grid simulation, upscaling results might be totally different. However, in the near-well region, we can show this upscaling procedure is almost independent on boundary conditions.

Let's study the application of the above technique for near-well upscaling. Considering a steady-state problem on a domain $\Omega$, which contains a well, with two different Dirichlet boundary conditions:

$$
\begin{cases}-\left(k_{x} \frac{\partial^{2} p}{\partial x^{2}}+k_{y} \frac{\partial^{2} p}{\partial y^{2}}\right)=q \delta(O) & (\mathrm{x}, \mathrm{y}) \in \Omega \\ p(x, y)=p_{R}(\mathrm{x}, \mathrm{y}) & (\mathrm{x}, \mathrm{y}) \in \Gamma_{\mathrm{R}}\end{cases}
$$

and

$$
\left\{\begin{array}{lr}
-\left(k_{x} \frac{\partial^{2} \tilde{p}}{\partial x^{2}}+k_{y} \frac{\partial^{2} \tilde{p}}{\partial y^{2}}\right)=\tilde{q} \delta(O) & (\mathrm{x}, \mathrm{y}) \in \Omega \\
\tilde{p}(x, y)=\tilde{p}_{R}(\mathrm{x}, \mathrm{y}) & (\mathrm{x}, \mathrm{y}) \in \Gamma_{\mathrm{R}}
\end{array}\right.
$$

where $k_{x}, k_{y}$ are permeabilities in $x$ and $y$ direction, the well is represented by a point sink/source and is located at the point $O, q$ or $\tilde{q}$ is the well flow rate.

We will show that the pressure behavior of these two solutions are generally very close in the well vicinity. Let $u=p-C \tilde{p}$ with $C=q / \widetilde{q}$, u should be the solution of the following problem:

$$
\begin{cases}-\left(k_{x} \frac{\partial^{2} u}{\partial x^{2}}+k_{y} \frac{\partial^{2} u}{\partial y^{2}}\right)=0 & (\mathrm{x}, \mathrm{y}) \in \Omega \\ u(x, y)=p(x, y)-C \tilde{p}_{R}(\mathrm{x}, \mathrm{y}) & (\mathrm{x}, \mathrm{y}) \in \Gamma_{\mathrm{R}}\end{cases}
$$

This problem corresponds to a steady-state flow on $\Omega$ without any well inside.

In Eq. (3) and Eq. (4), we have a well singularity. The pressure and flux in the well vicinity is large and tends to infinite, when the distance of a point to the well tends to zero. However, Eq. (5) is a regular problem, we have not any well singularity inside the domain $\Omega$. Like solution splitting in homogeneous media (cf. [7]), the solution of Eq. (3) can be split into a singular part $C \tilde{p}$ and a regular part $u$ :

$$
p=\widetilde{C p}+u
$$

In the well vicinity, the singular part is usually dominating, and the contribution of the regular part can be neglected. This is particularly true for calculation of pressure differences or pressure derivatives (flux). If we neglect the contribution of the regular solution $u$, transmissibilities determined with Eq. (3) or Eq. (4) are almost identical, as:

$$
T_{i j}=-\frac{F_{i j}}{p_{j}-p_{i}}=-\frac{C \widetilde{F}_{i j}+F_{u}}{C \tilde{p}_{j}-C \widetilde{p}_{i}+u_{j}-u_{i}} \approx-\frac{C \widetilde{F}_{i j}}{C \widetilde{p}_{j}-C \widetilde{p}_{i}}=-\frac{\widetilde{F}_{i j}}{\widetilde{p}_{j}-\widetilde{p}_{i}}=\widetilde{T}_{i j}
$$

where $\widetilde{F}_{i j}$ is the flux across the interface $\Gamma_{i j}$ for Eq. (4), $F_{u}$ is the flux across the interface $\Gamma_{i j}$ corresponding to the singular solution Eq. (5), $\widetilde{p}_{i}, \widetilde{p}_{j}$ are equivalent coarse gridblock pressures of the Eq. (4), and $u_{i}, u_{j}$ are the equivalent coarse gridblock pressures of the Eq. (5).

To minimize the contribution of the regular solution in the well vicinity, coarse gridblocks should be relatively close to the well, or the simulation domain $\Omega$ should be large enough with respect to considered coarse gridblock size. Larger the simulation domain $\Omega$, more accurate near-well upscaling results. Size of a simulation domain influence directly upscaling accuracy. In practice, even using a small simulation domain (a distance of two coarse gridblock size from the well) can generally improve simulation results. 
As discussed above, we can use either upscaled transmissibility $T_{i j}$ and $\widetilde{T}_{i j}$, which are not very different, for coarse grid flow simulation with a two-points scheme in the well vicinity. Now, let's study fluw truncation errors with this calculation. Assuming upscaled transmissibility $T_{i j}$ is used for coarse grid simulation, the flux approximation error in the well vicinity is estimated by:

$$
\varepsilon=\hat{F}_{i j}-\left(-T_{i j}\left(\hat{p}_{j}-\hat{p}_{i}\right)\right)
$$

where $\hat{F}$ corresponds to the real flied flux and $\hat{p}$ corresponds to the real field pressure. As discussed above, field pressure and flux in the near-well region can be split by:

$$
\hat{p}=C p+u
$$

and

$$
\hat{F}=C F+F_{u}
$$

where $p$ is the solution of Eq. (3), $u$ is a regular solution in the well vicinity, $F$ and $F_{u}$ represent flux corresponding to $p$ and $u$. Therefore, the flux calculation error is given by:

$$
\begin{aligned}
\varepsilon & =C F+F_{u}-\left(-T_{i j}\left(C p_{j}+u_{j}-C p_{i}-u_{i}\right)\right) \\
& =C F-\left(-T_{i j}\left(C p_{j}-C p_{i}\right)\right)+F_{u}-\left(-T_{i j}\left(u_{j}-u_{i}\right)\right) \\
& =F_{u}-\left(-T_{i j}\left(u_{j}-u_{i}\right)\right) \\
& =\varepsilon_{u}
\end{aligned}
$$

where $\varepsilon_{u}$ is the error of flux approximation on the regular solution. As singular solution is dominating in the well vicinity, if we neglect approximation error $\varepsilon_{u}$ for the regular solution, we can consider that the proposed near-well upscaling gives good flux approximations.

To get accurate near-well upscaling results, it is necessary having accurate calculations of equivalent coarse gridblock permeability and flux across a coarse gridblock edge. Calculation techniques for these terms will be presented directly for 3D problem with advanced wells in the next session.

For well modelling on coarse gridblocks, we need also upscale numerical well index (WI) on wellblocks. In fact, numerical $W I$ on a coarse gridblock $i$ can be determined as follows:

$$
W I_{i}=\frac{q_{i}}{p_{i}-p_{w i}}
$$

where $q_{i}$ is the well flow rate on the coarse gridblock $i, p_{i}$ is the wellblock pressure and $p_{w i}$ is the well flowing pressure on the gridblock $i$. Like transmissibility upscaling, numerical $W I$ calculated by the above formula is not sensitive to boundary conditions.

\section{Near-well upscaling in 3D for advanced wells on CPG gridblocks}

Two-points flux approximation scheme and upscaling procedure in $3 D$

A two-point flux approximation scheme can always by written as Eq. (1) for 3D flow modelling. Like the problem in 2D, the two-points scheme is generally inaccurate. But it can be considered adequate in the well vicinity. The key point of possible using a two-points scheme in the well vicinity is the determination of transmissibility using a near-well pressure solution through near-well upscaling procedure.

For reservoir modelling, the fine grid, issue from a geostatistical model to define heterogeneity, is generally Cartesian, and the coarse grid used for flow simulation, is usually CPG. For near-well upscaling, it is necessary to select a simulation zone for fine grid simulation and a transmissibility modification zone on coarse gridblocks. These two zones are not necessarily the same, but the transmissibility modification zone should be contained in the simulation zone. It is recommended that these zones contains at least two coarse gridblocks (including the well block) in each direction from the well. Although boundary conditions have less impact on near-well upscaling, it is always preferable using boundary conditions close to the reality. When reservoir information is not provided on 
boundary simulation zone for fine grid simulation, Dirichlet condition is preferred. The fine gird simulation should take into account existence of faille, pinchout etc. described by the geostatistical model. Once a near-well solution is obtained with fine grid simulation, we can calculate equivalent coarse gridblock pressures, flux and well flow rate on a coarse gridblock. With these data, equivalent transmissivility and numerical WI on wellblock can be determined with Eq. (2) and Eq. (9).

Calculations of equivalent coarse gridblock interface flux, coarse gridblock pressure and well flow rate on coarse blocks are described in following sections. Among these equivalent parameters, accurate calculation of flux on coarse block interface is the most important, as the numerical scheme is based on control-volume approach for accurate flux approximation.

\section{Calculation of flux on coarse gridblock interface}

The main difficulty of flux upscaling (calculation of equivalent flux on coarse gridblock interface) is the nonsuperposition between coarse and fine gridblocks. Assuming reservoir coarse gridblocks are rectilinear in vertical direction, an interface between two coarse gridblocks is constructed by a polygon with maximum 6 edges in 3D (Figure 2) (cf. [6]). A polygon can be decomposed by triangles. So, we need to present a technique for flux calculation on a triangle.

To calculate intersections between a triangle and fine gridblocks, marching cube method (cf. [11]) is used. The marching cube method, which is originally developed in medicine for $3 \mathrm{D}$ visualization, allows the calculation of intersections between a plane and a structure grid. In this application, intersections between a cut plane, which is the plane determined by a triangle, and fine gridblocks are first determined. Then, we calculate intersections of the triangle with respect to fine gridblocks on the cut plane, which is a $2 \mathrm{D}$ problem. This calculation will decompose a triangle $T$ into $N$ small triangles (Figure 3), and each triangle $T_{i}(i=1, \ldots, N)$ is entirely contained in a fine gridblock. The flux on the triangle $T$ is the sum of flux on small triangles:

$$
F=\int_{T} v_{n}(S) d \sigma_{s}=\sum_{i=1}^{N} \int_{T_{i}} v_{n}(S) d \sigma_{s}=\sum_{i=1}^{N} F_{i}
$$

where $v_{n}$ is the velocity on the triangular surface in the normal direction and $S$ is a point on this triangle. If we assume velocity is linear inside each fine gridblock, the flux on a small triangle $T_{i}$ can be calculated by:

$$
F_{i}=v_{n}\left(S_{c i}\right) \operatorname{mes}\left(T_{i}\right)
$$

where $S_{c i}$ is the center of the triangle $T_{i}$, mes $\left(T_{i}\right)$ represents area of the triangle $T_{i}$.

To determine the velocity at the triangle center $S_{c i}$, Pollock's method (cf. [14]) can be used. For instance, the velocity in $x$-direction at a point $S$ in a Cartesian fine gridblock is expressed by:

$$
v_{x}=B_{x}\left(x_{s}-x_{1}\right)+v_{x 1}
$$

with

$$
B_{x}=\left(v_{x 2}-v_{x 1}\right) / \Delta x_{i 0}
$$

where $v_{x l}, v_{x 2}$ are velocities on the left and right facets of the gridblock in $x$-direction, $x_{s}$ is the $x$-coordinate of the point $S, x_{1}$ is the $x$-coordinate of the left facet in $x$-direction, and $\Delta x_{i 0}$ is the gridblock size in $x$-direction.

Therefore, we can calculate flux on each coarse block facet by summing flux on decomposed triangles.

\section{Calculation of equivalent pressure on coarse gridblock} constant:

Equivalent coarse gridblock pressure can be calculated with the following formula, assuming porosity is

$$
\bar{p}=\frac{1}{V} \int_{V} p(x, y, z) d V
$$

where $\bar{p}$ is the equivalent pressure on this block, $V$ is the volume of a coarse gridblock. 
To calculate numerically the integral on a volume $V$, the geometry of the coarse gridblock is first transformed to a standard cube. Gauss-Legendre formula is then applied on the standard cube for numerical integral calculation. A detailed description is given in [6].

\section{Calculation of well flow rate on coarse gridblock}

Fine grid simulation can give well flow rate on each fine gridblock containing the well. As well geometry such as length or wellbore radius on each fine wellblock is known, flow rate distribution along the well can be determined. With these information, we can redistribute flow rate along the well according to its intersections with coarse CPG gridblocks.

\section{Examples}

\section{Example 1.}

A horizontal well is located in a box reservoir as shown in Figure 4. This well is not parallel to the principal axes of the reservoir. Data of the reservoir and the well are given in Table 1. A CPG grid is generated for flow simulation as shown in Figure $4 \mathrm{~b}$. This CPG grid is distorted in horizontal plane, and it has a regular discretization in vertical direction with 5 layers. The thickness of each layer is $25 \mathrm{~m}$. This grid follows the well trajectory as shown in the figure.

Heterogeneity is generated on $100 \times 100 \times 50$ fine gridblocks with block size $15 \times 15 \times 2.5 \mathrm{~m}^{3}$. The mean logpermeability is 3 , and its variance is $\sigma^{2}=1$. Correlation length is $50 \mathrm{~m}$ in horizontal direction, and there is no correlation in vertical direction. Permeability ratio between vertical and horizontal direction is 0.2 . Fine grid simulation result is considered as reference solution. Simulations on coarse CPG grid are compared with the fine grid simulation. For coarse grid simulation, both block permeability upscaling and near-well upscaling techniques are used and compared. As permeability upscaling on distorted gridblock is not well developed in the literature, we use simply the power law to calculate block permeability:

$$
\bar{k}=\left(\frac{1}{N} \sum_{i=1}^{N} k_{i}^{\omega}\right)^{\frac{1}{\omega}}
$$

In this example, we consider particularly upscaling procedure using harmonic average with $\omega=-1$, geometric average with $\omega=0$, and arithmetic average with $\omega=1$. When using block permeability upscaling, Peaceman's method is used for the determination of numerical well index (WI). Relative errors of coarse grid simulation on bottom hole pressure drop, which is equivalent to relative errors on well performance, are presented in Table 2. It is found that errors are large with a variation from $16 \%$ to $80 \%$.

Based on block permeability upscaling, we modify results in the near-well region by using proposed nearwell up-scaling procedure. For near-well upscaling, a simulation domain, used for fine grid simulation, and a transmissibility modification area on coarse gridblocks should be defined. In this example, simulation domain contains all fine gridblocks with no flow boundary condition on top and bottom of the reservoir and Dirichlet conditions on lateral boundary. This boundary condition does not correspond to the real one, which is flow null everywhere on the closed box shape reservoir. Transmissibility modification area is shown in Figure 5 . Table 2 shows clearly that simulation results can be greatly improved if near-well transmissibilities are upscaled using proposed near-well upscaling procedure.

In this example, if only block permeabilities are upscaled, upscaling with geometrical average gives much better result than that with harmonic average (error of $16 \%$ instead of $80 \%$ ). However, if near-well upscaling is applied, all errors can be reduced to $1-2 \%$. Advantage of geometric average over harmonic average is not significant, if near-well upscaling procedure is applied. Accuracy of block permeability outside near-well transmissibility modification range is not very important. Even if upscaled block permeability is not convenient, well performance accuracy can be greatly improved by modifying transmissibility values in the well vicinity using the near-well upscaling procedure. This example shows the importance of using the near-well upscaling procedure.

Simulation result with the above near-well upscaling procedure can be improved again if a larger transmissibility modification area is used. For example, if we modify transmissibilities on whole coarse gridblocks, error can be reduced to $0.12 \%$. It has to be mentioned that in this example although boundary conditions used in upscaling do not correspond to the real case, excellent results are obtained on well performance calculation. 


\section{Example 2.}

In this example, we will test the impact of permeability variance and correlation length on coarse grid flow simulation accuracy. We use the same reservoir geometry and well configuration as shown in the previous example. But the reservoir heterogeneities are changed. Heterogeneities are generated on $30 \times 30 \times 15$ gridblocks with block sizes $50 \times 50 \times 8.333 \mathrm{~m}^{3}$. Transmissibility modification zone is the same as described in Figure 5. Block permeabilities outside this zone are upscaled with geometric average.

Permeability field is generated with a log-normal permeability distribution using an exponential variogram model. The mean log-permeability is 3 , and different contrasts of permeability are tested with variance $\sigma^{2}=0.2,0.5$, 1. and 2. First, we assume that correlation length is $50 \mathrm{~m}$ in horizontal direction and there is no correlation in vertical direction. Simulation results on both coarse grid and fin grid are presented in Table 3. It is found that all coarse grid simulation results are accurate enough and permeability contrast has not great impact on near-well upscaling accuracy.

Impact of correlation length is tested for permeability variance $\sigma^{2}=2$. Simulation results with correlation length $\mathrm{lc}=50 \mathrm{~m}, 100 \mathrm{~m}, 200 \mathrm{~m}, 500 \mathrm{~m}$ and $1000 \mathrm{~m}$ are presented in Table 3 . When correlation length is larger than gridblock size, upscaling becomes usually less accurate. But all results can be considered acceptable. For the case of $l_{c}=200 \mathrm{~m}$, error is relatively large $(2.7 \%)$. It has to be mentioned that this result is much better than that when nearwell upscaling is not applied. Without near-well upscaling, error reaches to $109 \%$ in this case. If we increase transmissibility modification area, error can be reduced. For example, error is reduced to $0.22 \%$, if transmissibilities are modified on the whole domain.

\section{Conclusions}

In this paper, near-well upscaling techniques are analysed. It is shown that near-well upscaling results are not very sensitive to boundary conditions applied to upscaling procedures. Flow in the near-well region can be split into a singular one and a regular one, and the singular flow is usually dominating. The reason of small effect of boundary conditions on near-well upscaling results is that the near-well upscaling procedure is based on a singular flow and this flow can catch well singular flow behavior. If approximation errors for regular flow are neglected, near-well upscaling procedure can be considered accurate. For the same reason, a two-points flux approximation scheme can be applied on distorted gridblocks in the well vicinity to catch singular flow.

Upscaling on distorted gridblocks is addressed. The main difficulty of upscalinig on distorted grid is the non-superposition between coarse and fine gridblocks. In this paper, upscaling of transmissibility from a fine grid Cartesian grid, issue from a geostatistical model, to a coarse CPG grid for flow simulation is particularly discussed. To overcome the difficulty of non-superposition between these two gridblocks, marching cube method is applied to calculate facet intersection. Flux upscaling on coarse grid facet is calculated using techniques developed for stream lines. Coarse block pressure is calculated using Gauss-Legendre numerical integration.

The near-well upscaling technique is developed with 3D CPG grid for advanced well modelling. Examples show the importance of using near-well upscaling procedure for coarse grid flow simulation. Using near-well upscaling technique, errors can generally be controlled within $2 \%$. Boundary conditions for fine grid simulation are not necessarily to be the same as real cases. Near-well upscaling technique is also validated for different permeability variances and correlation lengths. Results show that satisfied results can be obtained with a transmissibility modification zone defined by two gridblocks from the well. Using larger transmissibility modification zone can improve again accuracy. The near-well upscaling procedure can assure the coherence of well performance prediction by using coarse grid and fine grid simulations.

\section{Acknowledgements}

I'd like to thank Mr. Davy LAN-NANG-FAN for his valued contribution and assistance.

\section{References}

[1] Aavastmark, I., Barkve, T., Boe, O. and Mannseth, T. : "Discretization on Unstructured Grids for Inhomogeneous Anisotropic Media” SIAM J. Sci Comput., vol 19, No 5, 1998. pp1700-1736.

[2] Christie, M.A., Wallstrom, T.C., Durlofsky, L.J., Sharp, D.H. and Zou, Q. : “Effective Medium Boundary Conditions in Upscaling" procceding of $7^{\text {th }}$ Euro. Conf. on Math. Oil Rec., Baveno, Lago Maggiore, Italy, 58 Sept. 2000.

$8^{\text {th }}$ European Conference on the Mathematics of Oil Recovery - Freiberg, Germany, 3 - 6 September 2002 
[3] Ding, Y.: "Scaling-up in the Vicinity of Wells in Heterogeneous Field" paper SPE 29137 presented at the $13^{\text {th }}$ SPE Symposium on Reservoir Simulation, San Antonio, TX, 12-15 Feb. 1995.

[4] Ding, Y. : “A Generalized 3D Well Model for Reservoir Simulation”, SPEJ, Dec. 1996.

[5] Ding, Y. and Urgelli, D. : "Upscaling of Transmissibility for Field Scale Flow Simulation in Heterogeneous Media" SPE 38016 presented at the $14^{\text {th }}$ SPE Symposium on Reservoir Simulation, Dallas, TX, June 8-11. 1997.

[6] Ding, Y. , Lemonnier, P., Estebenet, T. and Magras, J-F. : “A Control-Volume Method for Flow Simulation in Well Vicinity for Arbitrary Well” SPEJ 5 (1), March, 2000.

[7] Ding, Y. and Jeannin, L. : "A Multi-Point Flux Approximation Scheme for Well Modelling in Reservoir Simulations" Comp. Geosciences, Oct. 2001. pp93-119.

[8] Durlofsky, L., Milliken, W.J. and Bernath, A. : "Scaleup in the Near-Well Region" SPEJ 5 (1), March 2000. pp110-117

[9] Edwards, M.G. and Rogers, C.F. : “A Flux Continuous Scheme for the Full Tensor Pressure Equation" proceeding of $4^{\text {th }}$ Euro. Conf. On Math. Of Oil Rec., Roros, Norway, 1994.

[10] Holden, L., Nielsen, B.F. and Sannan, S. : "Upscaling of permeability using global norms" procceding of $7^{\text {th }}$ Euro. Conf. on Math. Oil Rec., Baveno, Lago Maggiore, Italy, 5-8 Sept. 2000.

[11] Loresen, W.E. and Cline, H.E., : "Marching Cubes: A High Resolution 3D Surface Construction Algorithm", Comp. Graphics, Vol. 2, N4, July, 1987.

[12] Mascarenhas, O. and Durlofsky, L.J. : "Coarse Scale Simulation of Horizontal Wells in Heterogeneous Reservoirs" J. of Petr. Sci. \& Eng., 25, 2000. pp 135-147

[13] Muggeridge, A.H., Cuypers, M., Bacquet, C. and Baker, J.W.: "Scale-up of Well Performance for Reservoir Flow Simulation" Proceeding of the $10^{\text {th }}$ Euro. Sym. on Improved Oil Recovery, Brighton, UK $18-20$ August 1999.

[14] Pollock, D. : "Semi-analytical Computation of Path Lines for Finite-Difference Models", Vol. 26, N6, Ground Water, Nov-Dec. 1988.

[15] Romeu, R.K. and Noetinger, B. : "Calculation of Internodal Transmissibilities in Finite Difference Models of Flow in Heterogeneous Media”, Water Resources Research, vol. 31, n 4, Juin 1995. Pp943-959.

[16] Terpolilli, P. and Hontans, T. : "Boundary Effects in the Upscaling of Absolute Permeability - a New Approach" procceding of $7^{\text {th }}$ Euro. Conf. on Math. Oil Rec., Baveno, Lago Maggiore, Italy, 5-8 Sept. 2000.

[17] Urgelli, D. : “Upscaling of Transmissibility Applied to Corner-Point Geometry" paper SPE 52063 presented at the SPE Euro. Petro. Conf., The Hague, Oct. 1998. 
Table 1. Data for the slanted horizontal well in a box-shape reservoir (example 1)

$\begin{array}{lll}\text { Reservoir geometry }(\mathrm{m}) & : & \begin{array}{l}\text { length }=1500 ; \\ \text { width }=1500 ; \\ \text { thickness }=125\end{array} \\ & & \begin{array}{l}\text { mean }(\mathrm{log})=3 ; \\ \text { Permeability }\end{array} \\ & : & \sigma^{2}=1 . \\ \text { Wellbore radius }(\mathrm{cm}) & \mathrm{r}_{\mathrm{w}}=10.8 \\ \text { Total compressibility }(1 / \mathrm{bar}) & : & \mathrm{c}=1.1 \mathrm{E}-4 \\ \text { Viscosity }(\mathrm{cPo}) & : & 1 \\ \text { Well flow rate }\left(\mathrm{m}^{3} / \mathrm{d}\right) & : & 100 \\ \text { Duratio (day) } & : & 20\end{array}$

Table 2. Simulation results in example 1

\begin{tabular}{|c|c|c|c|}
\hline $\begin{array}{c}\text { Block permeability } \\
\text { upscaling technique }\end{array}$ & $\begin{array}{c}\text { Near-well } \\
\text { upscaling }\end{array}$ & $\begin{array}{c}\text { Well performance } \\
\text { (Field PI) }\end{array}$ & $\begin{array}{c}\text { Error } \\
(\%)\end{array}$ \\
\hline Fine grid simulation & & 27.05 & - \\
\hline Arithmetic average & No & 34.97 & -22.63 \\
\cline { 2 - 4 } & Yes & 26.60 & 1.72 \\
\hline \multirow{2}{*}{ Geometric average } & No & 23.31 & 16.05 \\
\cline { 2 - 4 } & Yes & 26.74 & 1.18 \\
\hline \multirow{2}{*}{ Harmonic average } & No & 15.00 & 80.32 \\
\cline { 2 - 4 } & Yes & 26.88 & 0.64 \\
\hline & In whole & 27.02 & 0.12 \\
& domain & & \\
\hline
\end{tabular}

Table 3. Simulation results in example 2

\begin{tabular}{|c|c|c|c|c|c|}
\hline $\begin{array}{l}\text { Permeability } \\
\text { variance } \sigma^{2}\end{array}$ & $\begin{array}{l}\text { Correlation } \\
\text { length lc }\end{array}$ & $\begin{array}{l}\text { Near-well } \\
\text { upscaling }\end{array}$ & $\begin{array}{l}\text { Find grid } \\
\text { simulation }\end{array}$ & $\begin{array}{l}\text { Coarse grid } \\
\text { simulation }\end{array}$ & $\begin{array}{c}\text { Error } \\
(\%)\end{array}$ \\
\hline 0.2 & $\mathrm{lc}=50 \mathrm{~m}$ & Yes & 3.73 & 3.76 & 0.71 \\
\hline 0.5 & $\mathrm{lc}=50 \mathrm{~m}$ & Yes & 3.48 & 3.49 & 0.39 \\
\hline 1.0 & $\mathrm{lc}=50 \mathrm{~m}$ & Yes & 4.14 & 4.16 & 0.39 \\
\hline \multirow{7}{*}{2.0} & $\mathrm{lc}=50 \mathrm{~m}$ & Yes & 4.24 & 4.26 & 0.45 \\
\hline & $1 \mathrm{c}=100 \mathrm{~m}$ & Yes & 1.94 & 1.98 & 1.99 \\
\hline & \multirow{3}{*}{$\begin{array}{l}\mathrm{lc}=200 \mathrm{~m} \\
\mathrm{lc}=200 \mathrm{~m} \\
\mathrm{lc}=200 \mathrm{~m}\end{array}$} & Yes & 2.18 & 2.24 & 2.70 \\
\hline & & No & 2.18 & 4.57 & 109.3 \\
\hline & & Whole domain & 2.18 & 2.18 & -0.22 \\
\hline & $\mathrm{lc}=500 \mathrm{~m}$ & Yes & 4.33 & 4.38 & 1.03 \\
\hline & $\mathrm{lc}=1000 \mathrm{~m}$ & Yes & 3.92 & 3.95 & 0.80 \\
\hline
\end{tabular}



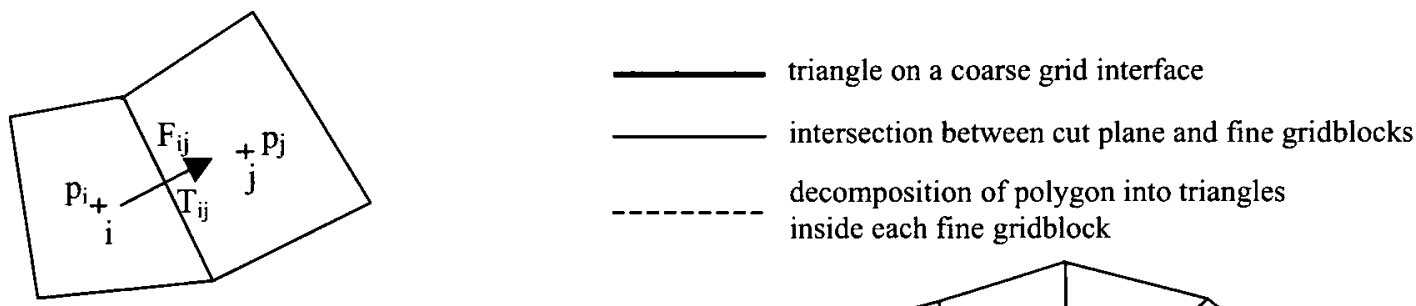

Figure 1--A two-points flux approximation scheme

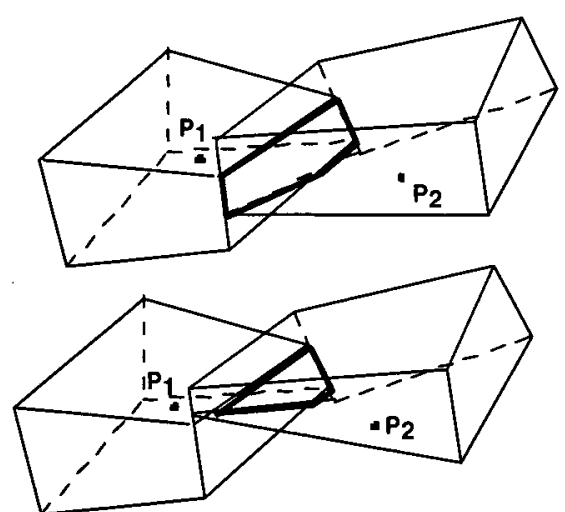

Figure 2--Example of interface between two coarse gridblocks

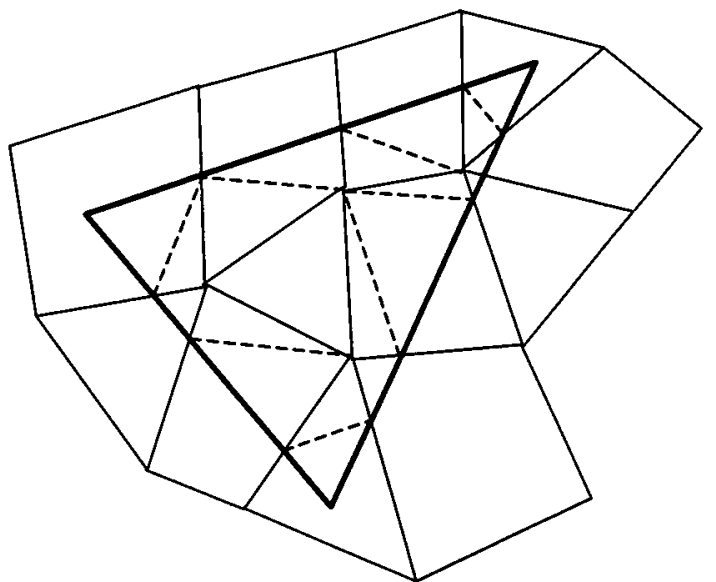

Figure 3--Intersection between a triangle and fine gridblocks on cut plane determined by the triangle

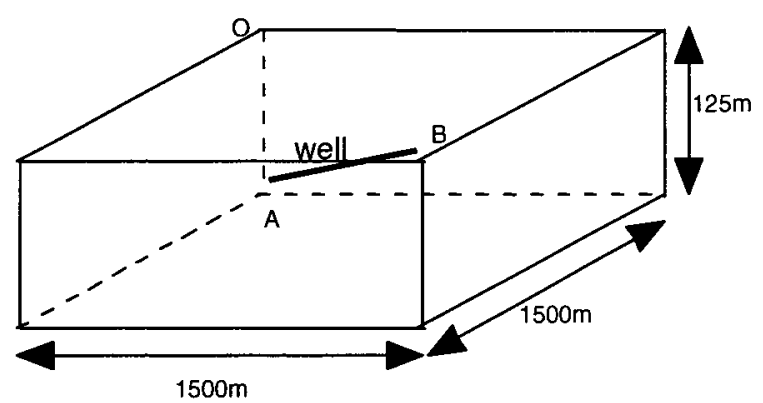

well extremity: $\quad A=(600,900,62) ; \quad B=(900,600,62)$

4a). Reservoir geometry and well position

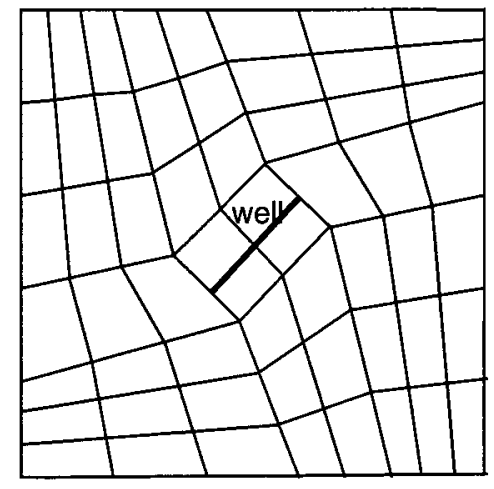

4b). Distorted gridblock in xy-plane

Figure 4--A horizontal well in a box-shape reservoir

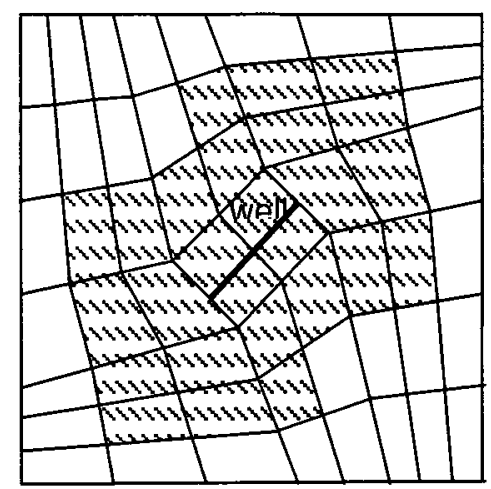

Figure 5--Transmissibility modification zone 